ELECTRONIC RESEARCH ANNOUNCEMENTS OF THE AMERICAN MATHEMATICAL SOCIETY

Volume 4, Pages 63-73 (September 15, 1998)

S $1079-6762(98) 00049-3$

\title{
THE NASH CONJECTURE FOR THREEFOLDS
}

\author{
JÁNOS KOLLÁR \\ (Communicated by Robert Lazarsfeld)
}

\begin{abstract}
Nash conjectured in 1952 that every compact differentiable manifold can be realized as the set of real points of a real algebraic variety which is birational to projective space. This paper announces the negative solution of this conjecture in dimension 3. The proof shows that in fact very few 3manifolds can be realized this way.
\end{abstract}

\section{INTRODUCTION}

In real algebraic geometry, one of the main directions of investigation is the topological study of the set of real solutions of algebraic equations. The first general theorem was proved in [Nash52] and later improved by [Tognoli73]. Their result says that every compact differentiable manifold is algebraic:

Theorem 1.1 ([Nash52, Tognoli73]). Let $M^{n}$ be a compact differentiable manifold. Then there are real polynomials $f_{i}\left(x_{1}, \ldots, x_{N}\right)$ such that their common zero set

$$
V\left(f_{1}, \ldots, f_{s}\right)(\mathbb{R}):=\left\{\mathbf{x} \in \mathbb{R}^{N}: f_{i}(\mathbf{x})=0 \forall i\right\} \subset \mathbb{R}^{N}
$$

is diffeomorphic to $M^{n}$.

Nash also considered further refinements of this result. To state these, we need to fix basic concepts, since the terminology in real algebraic geometry is, unfortunately, not at all uniform.

Definition 1.2. By a real algebraic variety I mean a variety given by real equations, as defined in most algebraic geometry books (see, for instance, [Shafarevich72, Hartshorne77]). This is different from the definition frequently used in real algebraic geometry which essentially considers only the germ of $X$ along its real points (cf. [BCR87]).

If $X$ is a real algebraic variety, then $X(\mathbb{R})$ denotes the set of real points of $X$ as a topological space and $X(\mathbb{C})$ denotes the set of complex points as a complex space. For all practical purposes we can identify $X$ with the pair $(X(\mathbb{C})$, complex conjugation).

The varieties $X=V\left(f_{1}, \ldots, f_{s}\right)$ constructed by Nash do not seem to have any special properties. Nash considered the question if (1.1) remains true for real algebraic varieties which are rational:

Received by the editors July 17, 1998.

1991 Mathematics Subject Classification. Primary 14P25.

(C) 1998 American Mathematical Society 
Definition 1.3. Let $X, Y$ be real algebraic varieties. We say that $X$ and $Y$ are birational if there is a birational map $\phi: X \rightarrow Y$ which is defined over $\mathbb{R}$. If $X$ is birational to $\mathbb{P}^{n}$, then $X$ is called rational over $\mathbb{R}$ or just rational. (Note that in many papers $X$ is called rational if $X(\mathbb{C})$ is birational to $\mathbb{P}^{n}$.)

Conjecture 1.4 ([Nash52, p. 421]). Let $M^{n}$ be a compact differentiable manifold. Then there is a smooth real algebraic variety $X^{n}$ such that $X$ is birational to $\mathbb{P}^{n}$ and $X(\mathbb{R})$ is diffeomorphic to $M^{n}$.

Unbeknownst to Nash, this question has been settled for surfaces much earlier.

Theorem 1.5 ([Comessatti14]). Let $S$ be a smooth real algebraic surface. Assume that $S$ is birational to $\mathbb{P}^{2}$ and $S(\mathbb{R})$ is orientable.

Then $S(\mathbb{R})$ is either a sphere or a torus.

In higher dimensions the conjecture of Nash remained open, and some partial results seemed to support the hope that it may hold in dimensions 3 and up. [Benedetti-Marin92] showed that for every 3-manifold $M^{3}$ there is a singular real algebraic variety $X^{3}$ such that $X$ is birational to $\mathbb{P}^{3}$ and $X(\mathbb{R})$ is homeomorphic to $M^{3}$. [Akbulut-King91] and [Mikhalkin97] showed that a weaker variant, the so-called "topological Nash conjecture" is true.

The aim of this paper is to announce a solution to the Nash conjecture in dimension 3. The result says that the Nash conjecture again fails completely. (The relevant basic concepts of 3-manifold topology are recalled in (1.11). See [Hempel76, Rolfsen76, Scott83] for more details.)

Main Theorem 1.6. Let $X$ be a smooth, projective, real algebraic 3-fold. Assume that $X$ is birational to $\mathbb{P}^{3}$ and that $X(\mathbb{R})$ is orientable. Then $X(\mathbb{R})$ is diffeomorphic to a 3-manifold

$$
M \# a \mathbb{R} \mathbb{P}^{3} \# b\left(S^{1} \times S^{2}\right) \quad \text { for some } a, b \geq 0,
$$

where $M$ is one of the following:

1. connected sum of lens spaces,

2. Seifert fibered,

3. $S^{1} \times S^{1}$-bundle over $S^{1}$ or a $\mathbb{Z}_{2}$-quotient of such.

4. finitely many other possibilities.

The proof establishes a tight connection between certain algebraic properties of $X(\mathbb{C})$ and geometric structures of $X(\mathbb{R})$. In some cases such a relationship has not been proved, and this accounts for the finitely many unknown cases. I believe, however, that there are no exceptions:

Conjecture 1.7. The cases (1.6), 3-4 do not occur.

Remark 1.8. The assumption that $X$ be birational to $\mathbb{P}^{3}$ can be weakened considerably. Namely, (1.6) also holds if we assume the following equivalent conditions

1. $X(\mathbb{C})$ is uniruled (that is, covered by rational curves),

2. $X$ has Kodaira dimension $-\infty$ (that is, $H^{0}\left(X, \mathcal{O}_{X}\left(m K_{X}\right)\right)=0$ for every $m \geq 1)$.

In this case $X(\mathbb{R})$ may have several connected components and each satisfies the conclusions of (1.6). I do not know what happens if some components of $X(\mathbb{R})$ are orientable and some are not. 
The orientability of $X(\mathbb{R})$ is not a crucial point for (1.6). The proof proceeds by a reduction argument. At each step we either get a nice description or we exhibit a special surface in $X(\mathbb{R})$. As far as I can tell, it is only an accident that all these special surfaces imply nonorientability. They also imply that $X(\mathbb{R})$ is not hyperbolic, and we obtain the following. (Again I conjecture that there are no exceptions.)

Theorem 1.9. There are only finitely many hyperbolic 3-manifolds (orientable or not) among the $X(\mathbb{R})$ where $X$ is a smooth, projective, real algebraic 3-fold such that $X(\mathbb{C})$ is uniruled.

A similar result was obtained in all dimensions by Viterbo, using stronger conditions on rational curves.

Theorem 1.10 ([Viterbo98]). Let $X$ be a smooth, projective, real algebraic variety of dimension $n \geq 3$. Assume that $H_{2}(X(\mathbb{C}), \mathbb{Z}) \cong \mathbb{Z}$ and that $X(\mathbb{C})$ is covered by rational curves $C_{\lambda}$ such that $\left[C_{\lambda}\right] \in H_{2}(X(\mathbb{C}), \mathbb{Z})$ is a generator.

Then $X(\mathbb{R})$ does not carry any metric with negative sectional curvature.

Definition 1.11. For relatively prime $0<q<p$ consider the action of $\mathbb{Z}_{p}$ on $S^{3} \sim\left(\left|x^{2}\right|+\left|y^{2}\right|=1\right) \subset \mathbb{C}^{2}$ given by $(x, y) \mapsto\left(e^{2 \pi i / p} x, e^{2 \pi i q / p} y\right)$. The quotient is a 3 -manifold called the lens space $L_{p, q}$.

A 3-manifold $M$ is called Seifert fibered if there is a morphism $f: M \rightarrow F$ to a topological surface such that every $P \in F$ has a neighborhood $P \in U \subset F$ such that $f: f^{-1}(U) \rightarrow U$ is fiber preserving diffeomorphic to one of the normal forms $f_{c, d}$ defined below.

Let $S^{1} \subset \mathbb{C}$ be the unit circle with coordinate $u$ and $D^{2} \subset \mathbb{C}$ the closed unit disc with coordinate $z$. For a pair of integers $c, d$ satisfying $0<c<d$ and $(c, d)=1$, define $f_{c, d}: S^{1} \times D^{2} \rightarrow D^{2}$ by $f_{c, d}(u, z)=u^{c} z^{d}$. $d$ is called the multiplicity of the fiber over 0 .

\section{ACKNOWLEDGMENTS}

I thank M. Bestvina, S. Gersten, G. Mikhalkin, M. Kapovich and S. Kharlamov for answering my numerous questions about 3-manifold topology and real algebraic geometry. I have received very helpful comments from V. Alexeev and M. Reid.

Partial financial support was provided by the NSF under grant number DMS9622394.

\section{OUtLine OF THE PROOF}

We utilize the theory of minimal models to investigate real algebraic threefolds. This approach is very similar in spirit to the one employed by [Comessatti14].

For algebraic threefolds over $\mathbb{C}$, the minimal model program (MMP for short) provides a very powerful tool. The method of the program is the following. (See [Kollár87, CKM88] or [Kollár-Mori98] for introductions.)

Starting with a smooth projective 3 -fold $X$, we perform a series of "elementary" birational transformations

$$
X=X_{0} \rightarrow X_{1} \rightarrow \cdots \rightarrow X_{n}=: X^{*}
$$

until we reach a variety $X^{*}$ whose global structure is "simple". (Neither the intermediate steps $X_{i}$ nor the final $X^{*}$ are uniquely determined by $X$.) In essence the minimal model program allows us to investigate many questions in two steps: first 
study the effect of the "elementary" transformations and then consider the "simple" global situation. In practice both of these steps are frequently rather difficult.

If $X$ is defined over $\mathbb{R}$, then there is a variant of the MMP where the intermediate varieties $X_{i}$ are also defined over $\mathbb{R}$. I refer to this as the MMP over $\mathbb{R}$. This suggests the following two step approach to understand the topology of $X(\mathbb{R})$ :

1. Study the topological effect of the "elementary" transformations.

2. Investigate the topology of $X^{*}(\mathbb{R})$.

A somewhat unpleasant feature of the theory is that the varieties $X_{i}$ are not smooth, but have so-called terminal singularities. This means that $X_{i}(\mathbb{R})$ is not necessarily a manifold. In developing the theory for real algebraic threefolds, we again have to understand the occurring terminal singularities. This is done in section 3 .

The heart of the method is the study of the "elementary" steps of the MMP over $\mathbb{R}$. In general these steps can be very complicated but, as it turns out, one can find reasonable conditions which ensure that the steps of the MMP can be described topologically.

In order to get an idea why this should be true, let us look at the MMP over $\mathbb{C}$. It is known by [Mori82, Cutkosky88] that the first nonhypersurface singularity that occurs is of the form

$$
\mathbb{C}^{3} / \mathbb{Z}_{2} \quad \text { where the action is } \quad(x, y, z) \mapsto(-x,-y,-z) .
$$

If the MMP over $\mathbb{R}$ behaves similarly, then we obtain $\mathbb{R}^{3} / \mathbb{Z}_{2}$ as a subset of the real points of some $X_{i} . \mathbb{R}^{3} / \mathbb{Z}_{2}$ is not orientable and it contains many copies of $\mathbb{R} \mathbb{P}^{2}$ which are 2 -sided. Thus we can hope to obtain a 2 -sided $\mathbb{R P}^{2}$ inside $X(\mathbb{R})$ as well. This leads to the working hypothesis

(*) If $X(\mathbb{R})$ does not contain a 2 -sided $\mathbb{R P}^{2}$, then all the steps of the MMP starting with $X$ are "simple".

Most 3 -manifolds do not contain a 2 -sided $\mathbb{R P}^{2}$, so this would be a quite nice result.

Unfortunately $(*)$ is not true. The reason is that a step of the MMP $X_{i} \rightarrow X_{i+1}$ may introduce a conjugate pair of points of the form $\mathbb{C}^{3} / \mathbb{Z}_{2}$. In this case $X_{i}(\mathbb{R})$ and the singular set of $X_{i}$ may be disjoint and they are unlikely to have any relationship. We may obtain a complicated real singular point at a later step of the program.

Fortunately, there are some indications that this does not happen. [Kawamata96] studied how singularities of the form $\mathbb{C}^{3} / \mathbb{Z}_{m}$ can appear during an MMP. His methods are easy to generalize to the real case and we get the following.

Proposition 2.1. If a real singularity of the form $\mathbb{C}^{3} / \mathbb{Z}_{m}$ appears at some stage of the $M M P$ over $\mathbb{R}$ then the real singularity $\mathbb{C}^{3} / \mathbb{Z}_{2}$ must have appeared at an earlier stage.

Attempts to extend the results of [Kawamata96] to more general singularities have been so far unsuccessful, but (2.1) points in the right direction. It turns out that there is a resonable list of steps where a "bad" singularity first appears. Moreover, each of these steps is associated with a surface of nonnegative Euler characteristic in $X(\mathbb{R})$. The details of this are explained in section 4 .

The next step is to study the topology of $X^{*}(\mathbb{R})$. So far we have not used that $X$ is birational to $\mathbb{P}^{3}$, but at this point this becomes crucial. The structure theory of [KoMiMo92] implies that a 3 -fold $X$ is uniruled iff $X^{*}$ falls in one of 3 classes: 
1. (Conic fibrations) There is a morphism $g: X^{*} \rightarrow S$ onto a surface defined over $\mathbb{R}$ such that the general fiber is a conic. Correspondingly there is a morphism $X^{*}(\mathbb{R}) \rightarrow S(\mathbb{R})$ whose general fibers are $S^{1}$ or empty.

2. (Del Pezzo fibrations) There is a morphism $g: X^{*} \rightarrow C$ onto a curve defined over $\mathbb{R}$ such that the general fiber is a Del Pezzo surface (these are special rational surfaces). Correspondingly, there is a morphism $X^{*}(\mathbb{R}) \rightarrow C(\mathbb{R})$ whose general fiber is a union of spheres, a torus or empty.

3. (Fano varieties) The anticanonical bundle of $X^{*}$ is ample.

The first two of these cases are discussed in section 5 . Section 6 contains some remarks about Fano varieties.

\section{Terminal singularities over $\mathbb{R}$}

Terminal singularities over $\mathbb{C}$ come in two families: hypersurface singularities and quotients of hypersurface singularities (see [Reid85] for a good introduction). Using the methods of [AGV85] it is easy to obtain normal forms for terminal hypersurface singularities over $\mathbb{R}$. $\left(f_{\geq m}\right.$ denotes any power series of multiplicity $\geq m$ and $f_{m}$ denotes its degree $m$ homogeneous part.)

Theorem 3.1 ([Kollár97, 2.8-10]). Assume that $F \in \mathbb{R}[[x, y, z, t]]$ defines a terminal singularity. Then, up to a sign and a coordinate change, $F$ can be brought to one of the following normal forms.

$c A_{n}: \quad x^{2} \pm y^{2}+f_{\geq n+1}(z, t), \quad(n \geq 0)$.

$c D_{4}: \quad x^{2}+f_{>3}(y, z, t), \quad\left(f_{3}\right.$ is not divisible by a square $)$.

$c D_{>4}: \quad x^{2}+y^{2} z+a y t^{r}+h_{\geq s}(z, t), \quad(a \in \mathbb{R}, r \geq 3, s \geq 4)$.

$c E_{6}: \quad x^{2}+y^{3}+y g_{\geq 3}(z, t)+h_{\geq 4}(z, t), \quad\left(h_{4} \neq 0\right)$.

$c E_{7}: \quad x^{2}+y^{3}+y g_{\geq 3}(z, t)+h_{\geq 5}(z, t), \quad\left(g_{3} \neq 0\right)$.

$c E_{8}: \quad x^{2}+y^{3}+y g_{\geq 4}(z, t)+h_{\geq 5}(z, t), \quad\left(h_{5} \neq 0\right)$.

Example 3.2. If $0 \in X$ is an isolated 3-dimensional singularity, then $X(\mathbb{R})$ is a cone over a surface near 0 . Consider for instance the singularities $X_{f}:=\left(x^{2}+\right.$ $\left.y^{2}+f(z, t)=0\right)$. Assume that $f(z, t)$ is negative on $m$ connected domains near the origin. Then $X_{f}(\mathbb{R})$ is a point for $m=0$, a cone over a torus for $m=1$ and a cone over $m$ disjoint spheres for $m>1$.

If $M$ is a 3-complex which is locally always like a cone over disjoint spheres, then its topological normalization $\bar{M}$ is a manifold and $M$ is obtained from $\bar{M}$ by pinching together finite collections of points.

Quotient singularities frequently have unexpected real forms. Consider for instance the $\mathbb{Z}_{4}$-action on $\mathbb{C}^{2}$ given by $(x, y) \mapsto(i x,-i y)$. There is an isomorphism

$$
\mathbb{C}^{2} / \mathbb{Z}_{4} \cong\left(u v-w^{4}=0\right) \subset \mathbb{C}^{3} \quad \text { given by } \quad u, v, w \mapsto x^{4}, y^{4}, x y .
$$

The singularity $\left(u v-w^{4}=0\right)$ has 3 different real forms:

$$
u v-w^{4}=0, \quad u^{2}+v^{2}-w^{4}=0 \quad \text { and } \quad u^{2}+v^{2}+w^{4}=0 .
$$

For 3-dimensional terminal singularities this does not happen. As Reid pointed out, this is closely related to the fact that the canonical divisor class generates the torsion subgroup of the local Picard group. We obtain the following classification. (Index $=n$ means that we take quotient by $\mathbb{Z}_{n}$ and weights $=(a, b, c, d)$ means that the action is $(x, y, z, t) \mapsto\left(\epsilon^{a} x, \epsilon^{b} y, \epsilon^{c} z, \epsilon^{d} t\right)$ where $\epsilon$ is a primitive $n$th root of unity. Note that in each case the action itself is not defined over $\mathbb{R}$ but the ring of invariants has a $\mathbb{C}$-basis consisting of monomials, which gives a real structure.) 
Theorem 3.3 ([Kollár97, 3.4]). Let $0 \in X$ be a 3-fold terminal nonhypersurface singularity over $\mathbb{R}$. Then $0 \in X$ is isomorphic over $\mathbb{R}$ to a singularity described by the following list:

\begin{tabular}{|c|l|c|l|l|}
\hline name & \multicolumn{1}{|c|}{ equation } & index & weights & condition \\
\hline$c A / 2$ & $x^{2} \pm y^{2}+f(z, t)$ & 2 & $(1,1,1,0)$ & \\
\hline$c A / n$ & $x y+f(z, t)$ & $n \geq 3$ & $(r,-r, 1,0)$ & $(n, r)=1$ \\
\hline$c A x / 2$ & $x^{2} \pm y^{2}+f_{\geq 4}(z, t)$ & 2 & $(0,1,1,1)$ & \\
\hline$c A x / 4$ & $x^{2} \pm y^{2}+f_{\geq 2}(z, t)$ & 4 & $(1,3,1,2)$ & \\
\hline$c D / 2$ & $x^{2}+f_{\geq 3}(y, z, t)$ & 2 & $(1,0,1,1)$ & \\
\hline$c D / 3$ & $x^{2}+f_{\geq 3}(y, z, t)$ & 3 & $(0,2,1,1)$ & $f_{3}(1,0,0) \neq 0$ \\
\hline$c E / 2$ & $x^{2}+y^{3}+f_{\geq 4}(y, z, t)$ & 2 & $(1,0,1,1)$ & \\
\hline
\end{tabular}

\section{The minimal model Program over $\mathbb{R}$}

In order to understand how $X(\mathbb{R})$ is obtained from $X^{*}(\mathbb{R})$ it is necessary to study the intermediate steps of the MMP. These steps are, unfortunately, not well understood even over $\mathbb{C}$. Thus we change point of view somewhat, and try to describe how a given $Y$ can be the target of one step $Y_{1} \rightarrow Y$. Again we run into problems, and the answer is not known even if $Y$ is smooth.

We thus try to describe only the "simplest" steps $Y_{1} \rightarrow Y$. To do this, we need to develop a measure of how to compare the exceptional divisors of different birational maps $Y_{i} \rightarrow Y$.

4.1. The hierarchy of exceptional divisors. Let $S$ be a smooth surface and $f_{1}: S_{1} \rightarrow S$ the blowup of a smooth point with exceptional curve $E_{1} \subset S_{1}$. Next blow up a point on $E_{1}$ to obtain $f_{2}: S_{2} \rightarrow S_{1}$ with exceptional curve $E_{2}$. The composite $f_{1} \circ f_{2}: S_{2} \rightarrow S$ has two exceptional curves which I denote by $E_{1}$ and $E_{2}$ by a slight abuse of notation. One can easily compute that the Jacobian of $f_{1} \circ f_{2}$ vanishes along $E_{1}$ to order 1 and along $E_{2}$ to order 2 . If we perform further blowups, we obtain curves with higher and higher order vanishing of the Jacobian along them.

Thus the order of vanishing of the Jacobian establishes a hierarchy of all exceptional curves, with the smallest order of vanishing corresponding to curves that appear after just one blowup.

A similar argument applies to blowups of smooth varieties in any dimension. Terminal singularities are essentially defined to make a similar hierarchy possible, but there are two problems. First, the correct analog of the order of vanishing of the Jacobian of $f: Z \rightarrow Y$ along an exceptional divisor $E \subset Z$ is only a positive rational number. It is called the disrepancy and it is denoted by $a(E, Y)$ (see [Kollár98a, 3.3] or [Kollár-Mori98, 2.25] for more details). Second, and this is more serious, if we have $f_{2}: Y_{2} \rightarrow Y_{1}$ and $f_{1}: Y_{1} \rightarrow Y$ with exceptional divisors $E_{2}$ and $E_{1}$, then we can only say that

$$
a\left(E_{2}, Y\right) \geq \frac{1}{m}\left(a\left(E_{1}, Y\right)+1\right)
$$

where $m$ is the index of $Y_{1}$ at $f_{2}\left(E_{2}\right)$. If $Y_{1}$ has index 1 at $f_{2}\left(E_{2}\right)$, then we are in good shape, but not in general. 
4.2. Gateways. Assume now that everything is over $\mathbb{R}$ and that $Y_{1}$ has index 1 along $Y_{1}(\mathbb{R})$. If we know that $E_{2}(\mathbb{R})$ is Zariski dense in $E_{2}(\mathbb{C})$, then $f_{2}\left(E_{2}\right)$ is a real point of $Y_{1}$.

This observation leads us to study those steps $X_{i} \rightarrow X_{i+1}$ of the MMP over $\mathbb{R}$ such that $K_{X_{i}}$ is Cartier along $X_{i}(\mathbb{R})$, but this fails for $X_{i+1}$. These are called gateway contractions in [Kollár98a], since they are the gateways through which the MMP can leave the category of "nice" varieties.

A lengthy case by case analysis produces a list of such gateways [Kollár98a, 8.2]. A study of each of these gateways is relatively straightforward, and in most cases there is a surface of nonnegative Euler characteristic in $X_{i}(\mathbb{R})$. The remaining cases are rather mild and we obtain the following.

Theorem 4.1 ([Kollár98a, 1.13]). Let $X$ be a smooth, projective, real algebraic 3fold such that $X(\mathbb{R})$ is orientable. Let $f_{i}: X_{i} \rightarrow X_{i+1}$ be any of the intermediate steps of the $M M P$ over $\mathbb{R}$ starting with $X$. Then the topological normalization $\overline{X_{i}(\mathbb{R})}$ (3.2) is a 3-manifold and the following is a complete list of possibilities for $f_{i}$ :

1. ( $\mathbb{R}$-trivial) $f_{i}$ is an isomorphism in a (Zariski) neighborhood of the set of real points.

2. ( $\mathbb{R}$-small) $f_{i}: X_{i}(\mathbb{R}) \rightarrow X_{i+1}(\mathbb{R})$ collapses a 1 -complex to points and there are small perturbations $\tilde{f}_{i}$ of $f_{i}$ such that $\tilde{f}_{i}: \overline{X_{i}(\mathbb{R})} \rightarrow \overline{X_{i+1}(\mathbb{R})}$ is a homeomorphism.

3. (smooth point blowup) $f_{i}$ is the inverse of the blowup of a smooth point $P \in$ $X_{i+1}(\mathbb{R})$.

4. (singular point blowup) $f_{i}$ is the inverse of a weighted blowup of a singular point $P \in X_{i+1}(\mathbb{R})$. Up to real analytic equivalence near $P$, there are two cases:

(a) $X_{i+1} \cong\left(x^{2}+y^{2}+g_{\geq 2 m}(z, t)=0\right)$ where $g_{2 m}(z, t) \neq 0, m \geq 1$ and $X_{i} \cong B_{(m, m, 1,1)} X_{i+1}$.

(b) $X_{i+1} \cong\left(x^{2}+y^{2}+g_{\geq 2 m+1}(z, t)=0\right)$ where $m \geq 1, z^{2 m+1} \in g$ and $z^{i} t^{j} \notin g$ for $2 i+j<4 m+2 ; X_{i} \cong B_{(2 m+1,2 m+1,2,1)} X_{i+1}$.

By repeatedly applying (4.1) to each step of the MMP, we can compare $X^{*}(\mathbb{R})$ and $X(\mathbb{R})$. This shows that it is sufficient to prove (1.6) for $X^{*}$.

Theorem 4.2 ([Kollár98a, 1.2]). Let $X$ be a smooth, projective, real algebraic 3fold and $X^{*}$ the result of the $M M P$ over $\mathbb{R}$. Assume that $X(\mathbb{R})$ is orientable.

Then $K_{X^{*}}$ is Cartier along $X^{*}(\mathbb{R}), \overline{X^{*}(\mathbb{R})}$ is a topological 3-manifold and $X(\mathbb{R})$ can be obtained from $\overline{X^{*}(\mathbb{R})}$ by repeated application of the following operations:

0. throwing away all isolated points of $\overline{X^{*}(\mathbb{R})}$,

1. taking connected sums of connected components,

2. taking connected sum with $S^{1} \times S^{2}$,

3. taking connected sum with $\mathbb{R P}^{3}$.

The operations (4.2), 2-3 appear even if $X^{*}$ is smooth.

Example 4.3. Let $X$ be a smooth 3 -fold over $\mathbb{R}$ and $0 \in X(\mathbb{R})$ a real point. Set $Y=B_{0} X$. Then $Y(\mathbb{R}) \sim X(\mathbb{R}) \# \mathbb{R P}^{3}$.

Let $X$ be a smooth 3 -fold over $\mathbb{R}$ and $D \subset X$ a real curve which has a unique real point $\{0\}=D(\mathbb{R})$. Assume furthermore that near 0 the curve is given by equations $\left(z=x^{2}+y^{2}=0\right)$. Let $Y_{1}$ be the blowup of $D$ in $X$. $Y_{1}$ has a unique singular point; 
let $Y$ denote the result of blowing it up. It is not hard to see that $Y$ is smooth and $Y(\mathbb{R}) \sim X(\mathbb{R}) \#\left(S^{1} \times S^{2}\right)$.

\section{Conic and Del Pezzo fibrations}

If $X^{*}$ is either a conic or a Del Pezzo fibration, then we obtain a map of $X^{*}(\mathbb{R})$ to a 2- or 1-dimensional space. One can try to use this map to get a geometric description of $X^{*}(\mathbb{R})$. It turns out that this is indeed possible, but the two cases require different methods. We start with the conic bundle case.

Let $Y$ be a real algebraic 3-fold and $f: Y \rightarrow S$ a conic fibration. Then $Y(\mathbb{R}) \rightarrow$ $S(\mathbb{R})$ is a map of a 3-complex to a 2-complex such that every fiber has dimension 1 . Moreover, $Y(\mathbb{R}) \rightarrow S(\mathbb{R})$ is a circle bundle over the complement of a 1-complex. It seems quite promising that $Y(\mathbb{R}) \rightarrow S(\mathbb{R})$ is a Seifert fibration. Nonetheless, one has to be more careful, as the following example shows.

Example 5.1. It is known that every 3 -manifold $M$ can be written as a degree 3 branched covering of $S^{3}$, branched along a knot $C \subset S^{3}$ (cf. [Rolfsen76, 10.G]). Let $S^{3} \rightarrow S^{2}$ be a Hopf fibration which is in general position with respect to $C$. The composite can be factored as $M \rightarrow F \rightarrow S^{2}$ where $F$ is a 2-complex and $M \rightarrow F$ has connected fibers. $M \rightarrow F$ has all the properties enumerated above, but it is not a Seifert fibration in general.

From this we see that in order to understand the topology of a conic fibration, one has to study the special fibers in detail.

Let $Y$ be a real projective 3 -fold with terminal singularities such that $K_{Y}$ is Cartier along $Y(\mathbb{R})$ and $\overline{Y(\mathbb{R})}$ is an orientable 3-manifold. Let $f: Y \rightarrow S$ be a rational curve fibration such that $-K_{Y}$ is $f$-ample. An elementary topological argument shows that one can factor $\overline{Y(\mathbb{R})} \rightarrow S(\mathbb{R})$ as

$$
\overline{Y(\mathbb{R})} \stackrel{\tilde{f}}{\rightarrow} F \rightarrow S(\mathbb{R})
$$

where $F$ is a 2-manifold with boundary, $\tilde{f}$ is surjective and has connected fibers. It is not hard to see that $\tilde{f}$ is an $S^{1}$-bundle over $\operatorname{Int} F$, except at finitely many points $P_{1}, \ldots, P_{s}$. At the boundary points of $F$ one can choose local coordinates such that $\tilde{f}$ is given as $(x, y, z) \mapsto\left(x^{2}+y^{2}, z\right)$. It remains to establish a connection between the algebraic $f$ and the topological $\tilde{f}$ near the special points $P_{i}$.

Theorem 5.2 ([Kollár98b, 1.9]). Notation as above. For each $P_{i}$ there is $m=$ $m\left(P_{i}\right) \geq 2$ such that

1. $\tilde{f}: \overline{Y(\mathbb{R})} \rightarrow F$ has a Seifert fiber of multiplicty $m$ above $P_{i}$, and

2. near $P_{i}, f$ is real analytically isomorphic to

$$
\left(\mathbb{P}_{x: y: z}^{2} \times \mathbb{A}_{s, t}^{2}\right) / \mathbb{Z}_{m} \supset\left(x^{2}+y^{2}-z^{2}=0\right) / \mathbb{Z}_{m} \rightarrow \mathbb{A}_{s, t}^{2} / \mathbb{Z}_{m},
$$

where $\mathbb{Z}_{m}$ acts by rotation with angle $2 \pi / m$ on $(s, t)$, it fixes $z$ and acts by rotation with angle $2 a \pi / m$ on $(x, y)$ for some $(a, m)=1$.

If $F$ has no boundary, then $\tilde{f}: \overline{Y(\mathbb{R})} \rightarrow F$ is a Seifert fibration. If $F$ does have boundary, then it is easy to see that $\overline{Y(\mathbb{R})}$ is a connected sum of lens spaces. This proves (1.6) in the conic fibration case.

Next we study Del Pezzo fibrations. Here we have a morphism $f: Y \rightarrow C$ such that $Y$ has only isolated singularities, $-K_{Y}$ is $f$-ample, $K_{Y}$ is Cartier at all real points, every fiber of $f$ is irreducible (over $\mathbb{R}$ ) and $\overline{Y(\mathbb{R})}$ is a 3 -manifold. 
As in the conic fibration case, the essential point is the analysis of the singular fibers.

Let $A \sim S^{1}$ be a connected component of $C(\mathbb{R})$ and $p_{1}, \ldots, p_{s} \in A$ the points (in cyclic order) over which $f$ is not smooth. For each $i$ pick a point $q_{i} \in\left(p_{i}, p_{i+1}\right)$. Then $Y_{q_{i}}:=f^{-1}\left(q_{i}\right)$ is a smooth Del Pezzo surface and $Y_{q_{i}}(\mathbb{R})$ is orientable. Thus by $(1.5) Y_{q_{i}}(\mathbb{R})$ is either $S^{1} \times S^{1}$ or a disjoint union of copies of $S^{2}$. Gluing 3manifolds along such surfaces is a relatively simple operation, thus one can expect to get a good description of $Y(\mathbb{R})$ by describing the pieces $Z_{i}:=\left(f^{-1}\left[q_{i-1}, q_{i}\right]\right)(\mathbb{R})$ for every $i$.

$f: Z_{i} \rightarrow\left[q_{i-1}, q_{i}\right]$ is a function whose only critical value is $p_{i}$. Thus $Z_{i}$ can be viewed as a regular neighborhood of the critical level set $\left(f^{-1}\left(p_{i}\right)\right)(\mathbb{R})$.

The complex projective surface $S_{i}:=f^{-1}\left(p_{i}\right)$ is a "singular Del Pezzo" surface which appears as a degeneration of smooth Del Pezzo surfaces. Quite a lot is known about such surfaces (see, for instance, [Keel-McKernan98, Manetti91, Manetti93, Reid94]). Unfortunately, a complete classification of such singular Del Pezzo surfaces is not feasible because of the combinatorial complexity of the problem.

It is nonetheless possible to use the methods of these authors to develop a rough topological description of the $S_{i}(\mathbb{R})$. As in [Kollár98c], the end result is that $\overline{Y(\mathbb{R})}$ is glued together from the following pieces:

1. $S^{1} \times S^{2}$ minus open balls,

2. lens space minus open balls,

3. solid torus minus open balls,

4. interval bundle over a torus or a Klein bottle.

It is not hard to see that all such manifolds belong to one of the cases (1.6.1-3). This proves (1.6) in the Del Pezzo fibration case.

\section{Speculations}

6.1. Fano threefolds. In order to complete the proof of (1.6), we still have to deal with the case when $X^{*}$ is a Fano 3 -fold. There is a complete list of smooth Fano 3 -folds [Iskovskikh80]. It would be interesting to determine the possible topological types of smooth real Fano 3-folds. Unfortunately this may not be easy. For instance it is not known if $Y(\mathbb{R})$ can be hyperbolic for a smooth degree 4 hypersuface $Y \subset \mathbb{P}^{4}$. (The case of degree 4 surfaces $S \subset \mathbb{P}^{3}$ was treated in [Kharlamov76].)

In general it is known that there are only finitely many families of singular Fano varieties in dimension 3 [Kawamata92], but there is no explicit list. From this we conclude that as $Y$ runs through all singular Fano 3-folds, we obtain only finitely many topological spaces $Y(\mathbb{R})$ up to homeomophism. The methods of [Kollár93] can be used to derive an explicit upper bound. (I have not computed the resulting bound; it is probably something like $10^{10^{10}}$.)

This concludes the proof of (1.6).

Many Fano 3-folds are birational to conic or Del Pezzo fibrations, and this could be used to understand the topology of their real part. In other cases there is frequently a fibration whose general fibers are elliptic curves. It should be possible to develop a topological theory of real elliptic fibrations. Unfortunately I do not know a general result which asserts that such fibrations always exist. 
6.2. Higher-dimensional Nash conjecture. It is natural to consider the Nash conjecture in higher dimensions as well. The minimal model program is still conjectural in dimensions above 3 and it is very unlikely that terminal singularities or the steps of the program will ever be completely described. Nonetheless, (1.9) and (1.10) suggest that the following may be true:

Conjecture 6.1. Let $X$ be a smooth, projective, real algebraic variety of dimension $n \geq 3$. Assume that $X(\mathbb{C})$ is uniruled. Then $X(\mathbb{R})$ does not carry any metric with negative sectional curvature.

One can even go further and pose the following question, which is open even in dimension 3 .

Question 6.2. Let $X$ be a smooth, projective, real algebraic variety of dimension $n \geq 3$. Assume that $X(\mathbb{R})$ does carry a metric with negative sectional curvature. Is it true that $X$ is of general type?

6.3. The nonprojective Nash conjecture. For the minimal model program to work it is essential to have projective varieties. It would be interesting to know what happens for proper but nonprojective varieties. There are reasons to believe that the answer may be quite different from the projective case.

\section{REFERENCES}

Akbulut-King91. S. Akbulut and H. King, Rational structures on 3-manifolds, Pacific J. Math. 150 (1991), 201-204. MR 93c:57028

AGV85. V. I. Arnold, S. M. Gusein-Zade and A. N. Varchenko, Singularities of Differentiable Maps I-II, Birkhäuser 1985, 1988. MR 86f:58018; MR 89g:58024

Benedetti-Marin92. R. Benedetti and A. Marin, Déchirures de variétés de dimension trois, Comm. Math. Helv. 67 (1992), 514-545. MR 94d:57036

BCR87. J. Bochnak, M. Coste and M-F. Roy, Géométrie algébrique réelle, Springer, 1987. MR 90b: 14030

CKM88. H. Clemens, J. Kollár and S. Mori, Higher Dimensional Complex Geometry, Astérisque 166 (1988). MR 90j:14046

Comessatti14. A. Comessatti, Sulla connessione delle superfizie razionali reali, Annali di Math. 23(3) (1914), 215-283.

Cutkosky88. S. D. Cutkosky, Elementary contractions of Gorenstein threefolds, Math. Ann. 280 (1988), 521-525. MR 89k: 14070

Hartshorne77. R. Hartshorne, Algebraic Geometry, Springer, 1977. MR 57:3116

Hempel76. J. Hempel, 3-manifolds, Princeton Univ. Press, 1976. MR 54:3702

Iskovskikh80. V. A. Iskovskikh, Anticanonical models of three-dimensional algebraic varieties, J. Soviet Math. 13 (1980), 745-814. MR 81i:14026b

Kawamata92. Y. Kawamata, Boundedness of $\mathbb{Q}$-Fano threefolds, Proc. Int. Conf. Algebra, Contemp. Math. vol. 131 (1992), 439-445. MR 93g:14047

Kawamata96. Y. Kawamata, Divisorial contractions to 3-dimensional terminal quotient singularities, in Higher dimensional complex varieties (Trento, 1994), de Gruyter, (1996), 241-246. MR 98g:14005

Keel-McKernan98. S. Keel and J. McKernan, Rational curves on quasi-projective varieties, Mem. AMS (to appear). CMP 98:09

Kharlamov76. V. Kharlamov, The topological type of non-singular surfaces in $R P^{3}$ of degree four, Funct. Anal. Appl. 10 (1976), 295-305. MR 56:8584

Kollár87. J. Kollár, The structure of algebraic threefolds - an introduction to Mori's program, Bull. AMS 17 (1987), 211-273. MR 88i:14030

Kollár93. J. Kollár, Effective Base Point Freeness, Math. Ann. 296 (1993), 595-605. MR 94f: 14004

Kollár96. J. Kollár, Rational Curves on Algebraic Varieties, Springer-Verlag, Ergebnisse der Math. vol. 32, 1996. MR 98c:14001 
Kollár97. J. Kollár, Real Algebraic Threefolds I. Terminal Singularities, Collectanea Math. (to appear).

Kollár98a. J. Kollár, Real Algebraic Threefolds II. Minimal Model Program, J. AMS (to appear).

Kollár98b. $\quad$ J. Kollár, Real Algebraic Threefolds III. Conic Bundles (preprint).

Kollár98c. J. Kollár, Real Algebraic Threefolds IV. Del Pezzo Fibrations (in preparation).

KoMiMo92. J. Kollár, Y. Miyaoka and S. Mori, Rationally Connected Varieties, J. Alg. Geom. 1 (1992), 429-448. MR 93i:14014

Kollár-Mori98. J. Kollár and S. Mori, Birational geometry of algebraic varieties, Cambridge Univ. Press, 1998 (to appear).

Manetti91. M. Manetti, Normal degenerations of the complex projective plane, J. f.r.u.a. Math. 419 (1991), 89-118. MR 92f:14028

Manetti93. M. Manetti, Normal projective surfaces with $\rho=1, P_{-1} \geq 5$, Rend. Sem. Mat. Univ. Padova 89 (1993), 195-205. MR 94k:14027

Mikhalkin97. G. Mikhalkin, Blowup equivalence of smooth closed manifolds, Topology, 36 (1997), 287-299. MR 98f:57046

Mori82. S. Mori, Threefolds whose Canonical Bundles are not Numerically Effective, Ann. of Math. 116 (1982), 133-176. MR 84e:14032

Nash52. J. Nash, Real algebraic manifolds, Ann. Math. 56 (1952), 405-421. MR 14:403b

Reid85. M. Reid, Young person's guide to canonical singularities, in Algebraic Geometry, Proc. Symp. Pure Math. vol. 46, pp. 345-414. MR 89b:14016

Reid94. M. Reid, Nonnormal Del Pezzo surfaces, Publ. RIMS Kyoto Univ. 30 (1994), 695-728. MR 96a:14042

Rolfsen76. D. Rolfsen, Knots and links, Publish or Perish, 1976. MR 58:24236, MR 95c:57018

Scott83. P. Scott, The geometries of 3-manifolds, Bull. London Math. Soc., 15 (1983), 401-487. MR 84m:57009

Shafarevich72. R. I. Shafarevich, Basic Algebraic Geometry (in Russian), Nauka, 1972. English translation: Springer, 1977, 2nd edition, 1994. MR 51:3162; MR 56:5538; MR 95m:14001; MR 95m:14002

Tognoli73. A. Tognoli, Su una congettura di Nash, Ann. Scuola Norm. Sup. Pisa 27 (1973), 167-185. MR 53:434

Viterbo98. $\quad$ C. Viterbo, (personal communication).

University of Utah, Salt Lake City, UT 84112

E-mail address: kollar@math.utah.edu 